Please do not remove this page

RMIT

UNIVERSITY

\title{
Reduced cost photonic instantaneous frequency measurement system
}

Sarkhosh, Niusha; Emami, Hossein; Bui, Lam; Mitchell, Arnan

https://researchrepository.rmit.edu.au/esploro/outputs/9921861315901341/filesAndLinks?institution=61RMIT_INST\&index=null

Sarkhosh, N., Emami, H., Bui, L., \& Mitchell, A. (2008). Reduced cost photonic instantaneous frequency measurement system. IEEE Photonics Technology Letters, 20(18), 1521-1523.

https://doi.org/10.1109/LPT.2008.927895

Published Version: https://doi.org/10.1109/LPT.2008.927895

Repository homepage: https://researchrepository.rmit.edu.au

(C)2008 IEEE. Personal use of this material is permitted. However, permission to reprint/republish this material for advertising or promotional purposes or for creating new collective works for resale or redistribution to servers or lists, or to reuse any copyrighted component of this work in other works must be obtained from the IEEE.

Downloaded On 2023/04/27 00:42:16 +1000 


\title{
Reduced Cost Photonic Instantaneous Frequency Measurement System
}

\author{
Niusha Sarkhosh, Member, IEEE, Hossein Emami, Lam Bui, Member, IEEE, and Arnan Mitchell, Member, IEEE
}

\begin{abstract}
A wideband photonic instantaneous frequency measurement system is proposed and practically demonstrated. This system employs only a low-frequency inexpensive photodetector and thus the system cost is reduced.
\end{abstract}

Index Terms-Frequency measurement, microwave photonics.

\section{INTRODUCTION}

I NSTANTANEOUS frequency measurement (IFM) is important for modern radar warning receivers, providing initial threat classification of incoming signals and suggesting frequency ranges to focus processing resources.

Traditional IFM receivers have been implemented simply using interferometers formed from hybrid couplers and delay lines [1][2]. The bandwidth of such implementations can be limited due to unwanted radiation and dispersion occurring inside radio-frequency (RF) devices [3], [4].

On the other hand, microwave photonics has been suggested as a means of increasing the bandwidth of signal processing systems [5]-[8]. Broadband, low-noise optical modulators up-convert the RF signal to the optical domain where it is photonically processed and transmitted via optical fiber. Broadband photodetectors then down-convert it to the RF domain.

Photonics could offer advantages for broadband IFM systems. A recent photonics IFM was demonstrated using broadband high-performance photodetectors. However, banks of these devices would be prohibitively expensive [5].

In this letter, an alternate photonic IFM approach using lowcost low-frequency photodetectors is proposed and practically demonstrated, measuring frequencies from 1 to $10 \mathrm{GHz}$.

\section{IFM CONCEPT}

Fig. 1 shows a possible IFM system [1][2]. An RF tone is divided into two equal portions. One portion is delayed relative to the other by time $\tau$. The two RF tones are then multiplied together and the result is low-pass filtered. The output is a voltage proportional to $(1) /(4) V_{0}^{2} \cos \Omega \tau$, which varies with input RF frequency. This system can, therefore, be used to achieve a lowcost wideband IFM receiver; however, practical implementation of such a system would require broadband delays and mixers which can be challenging in the RF domain.

Manuscript received April 2, 2008; revised May 28, 2008.

The authors are with the Micro Electronics and Material Technology Center, School of Electrical and Computer Engineering, Royal Melbourne Institute of Technology, Melbourne, Victoria 3001, Austalia (e-mail: niusha.sarkhosh@rmit.edu.au).

Color versions of one or more of the figures in this letter are available online at http://ieeexplore.ieee.org.

Digital Object Identifier 10.1109/LPT.2008.927895

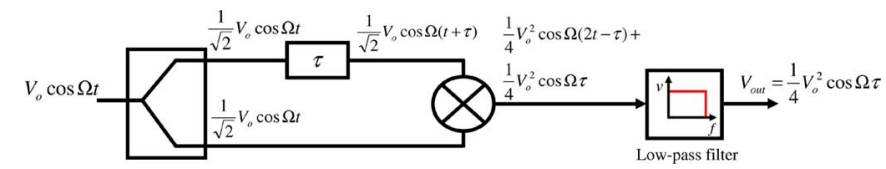

Fig. 1. Block diagram of an electrical IFM system.

\section{PHOTONIC IFM}

The system of Fig. 1 exhibits direct current (dc) output which is proportional to the RF frequency to be measured. Photonics can enable a broad frequency detection range and low-cost photodetectors could be used to reduce the total system cost.

\section{A. Photonic IFM System Configuration}

Fig. 2 shows the experimental setup of the proposed photonic IFM system. An RF signal generator produces a single RF tone which is divided equally into two portions using a Wilkinson power divider feeding two arms of the IFM system. These two arms are labelled the optical path and the RF path on Fig. 2. The $\mathrm{RF}$ tone in the optical path is input to a Mach-Zehnder modulator (MZM1) biased at quadrature $\left(V_{q}\right)$. MZM1 modulates an optical carrier with wavelength of $\lambda_{1}$ produced by a laser diode. The modulated carrier then traverses a fiber patch cord and experiences an optical delay. The second portion of the RF tone in the RF path is delayed using a length of co-axial cable, and is input to a second modulator (MZM2) (biased at $V_{q}$ ) modulating the optical carrier a second time. The twice-modulated signal is then detected by a photodetector. The output of the photodetector is then low-pass filtered and measured by a digital voltmeter. Having conceived a photonic IFM system configuration, we now develop a theoretical model to accurately predict the frequency dependence of the dc term of the photodetector output.

\section{B. Photonic IFM Model}

An optical carrier with angular frequency $\omega$ and power $P_{\circ}$ can be represented as $E(t)=E_{\circ} e^{j \omega t}$, where $E_{\circ}=\sqrt{P_{\circ}}$. Similarly, the RF signal with angular frequency $\Omega$ and power $P_{\mathrm{RF}}$ exerts an input voltage of $V_{\mathrm{RF}}(t)=V_{\circ} \cos \Omega t$, where $V_{\circ}=\sqrt{2 Z_{\mathrm{in}} P_{\mathrm{RF}}}$ across an input impedance of $Z_{\text {in }}$. For the system of Fig. 2, the RF signal is divided 50:50 using a broadband Wilkinson power divider; the input voltage to the modulator will thus be $V_{\text {in }}(t)=$ $(1) /(\sqrt{2}) V_{\mathrm{RF}}(t)$. The output of the modulator is

$$
E_{1}(t)=E(t) \sqrt{L_{1}} \cos \left[\frac{\pi\left(V_{\text {in }}+V_{B_{1}}\right)}{2 V_{\pi_{1}}}\right]
$$

where $L_{1}, V_{B_{1}}$, and $V_{\pi_{1}}$ are the modulator insertion loss, dc bias voltage, and half-wave voltage, respectively. Substituting 


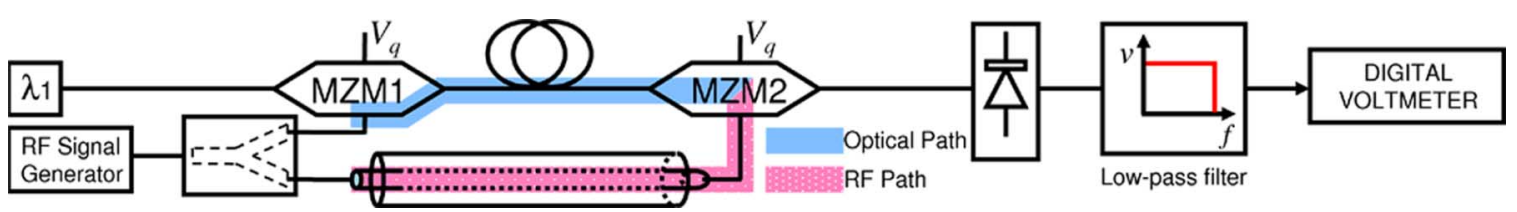

Fig. 2. Experimental setup of proposed photonic IFM.

the expression for $V_{\mathrm{in}}(t)$ and expanding the right-hand side of (1) into a Fourier series using Bessel functions of the first kind, the dc, fundamental, and harmonic components of the modulated optical carrier can be separated. Ignoring the harmonic frequency components, $E_{1}(t)$ can be approximated as

$$
\begin{aligned}
E_{1}(t) \approx E(t) \sqrt{L_{1}} \times[ & \cos \frac{\pi V_{B_{1}}}{2 V_{\pi_{1}}} J_{\circ}\left(\frac{\pi V_{\circ}}{2 V_{\pi_{1}}}\right) \\
& \left.-2 \sin \frac{\pi V_{B_{1}}}{2 V_{\pi_{1}}} J_{1}\left(\frac{\pi V_{\circ}}{2 V_{\pi_{1}}}\right) \cos \Omega t\right] .
\end{aligned}
$$

The output of MZM1 becomes the input to MZM2. This is modulated by the same input RF signal delayed by a differential time $(\tau)$ with respect to the optically carried RF signal. Since the modulating signal is attenuated and delayed by the co-axial cable in a nontrivial way, it should be represented as $M(\Omega) \cos (\Omega t+\phi(\Omega))$, where $M(\Omega)$ is the frequency-dependent amplitude response of the co-axial cable and $\phi(\Omega)$ denotes the frequency-dependent phase response of the co-axial cable relative to the optical path. For brievity, we will use $M$ and $\phi$ instead of $M(\Omega)$ and $\phi(\Omega)$. Using (2) with $E_{1}(t)$ as input, the output of MZM2 is

$$
\begin{aligned}
E_{2}(t) \approx & E_{1}(t) \sqrt{L_{2}} \\
\times & \times\left[\cos \frac{\pi V_{B_{2}}}{2 V_{\pi_{2}}} J_{\circ}\left(\frac{\pi M V_{\circ}}{2 V_{\pi_{2}}}\right)\right. \\
& \left.\quad-2 \sin \frac{\pi V_{B_{2}}}{2 V_{\pi_{2}}} J_{1}\left(\frac{\pi M V_{\circ}}{2 V_{\pi_{2}}}\right) \cos (\Omega t+\phi)\right] .
\end{aligned}
$$

Assuming similar modulators are used and both are biased at quadrature, we have $V_{\pi_{1}}=V_{\pi_{2}}=V_{\pi}$ and the sine and cosine of bias terms of (3) are all equal to $(1) /(\sqrt{2})$. In addition, for $V_{\pi} \approx 5 \mathrm{~V}$ and small $V_{\mathrm{o}}$, the following inequalities also hold $\left(\pi V_{\circ}\right) /\left(2 V_{\pi}\right) \ll 1$ and $M<1$. Bessel terms of (3) can be approximated

$$
\begin{aligned}
& J_{\circ}\left(\frac{\pi V_{\circ}}{2 V_{\pi_{1}}}\right) \approx 1, \quad J_{\circ}\left(\frac{\pi M V_{\circ}}{2 V_{\pi_{2}}}\right) \approx 1, \\
& J_{1}\left(\frac{\pi V_{\circ}}{2 V_{\pi_{1}}}\right) \approx \frac{\pi V_{\circ}}{4 V_{\pi}}, \quad J_{1}\left(\frac{\pi M V_{\circ}}{2 V_{\pi_{2}}}\right) \approx \frac{\pi M V_{\circ}}{4 V_{\pi}} .
\end{aligned}
$$

Therefore, (3) can be written as

$$
\begin{aligned}
E_{2}(t) \approx & e^{j \omega t} E_{\circ}^{2} \sqrt{\frac{L_{1} L_{2}}{4}} \\
& \times\left[1-\frac{\pi V_{\circ}}{2 V_{\pi}} \cos \Omega t\right] \times\left[1-\frac{\pi M V_{\circ}}{2 V_{\pi}} \cos (\Omega t+\phi)\right] \\
= & e^{j \omega t}[A+B \cos \Omega t][C+D \cos (\Omega t+\phi)]
\end{aligned}
$$

where $A, B, C$, and $D$ are

$$
\begin{aligned}
& A=\sqrt[4]{\frac{L_{1} L_{2} E_{\circ}^{2}}{4}}, \quad B=-\sqrt[4]{\frac{L_{1} L_{2} E_{\circ}^{2}}{4}} \times \frac{\pi V_{\circ}}{2 V_{\pi}} \\
& C=\sqrt[4]{\frac{L_{1} L_{2} E_{\circ}^{2}}{4}}, \quad D=-\sqrt[4]{\frac{L_{1} L_{2} E_{\circ}^{2}}{4}} \times \frac{\pi M V_{\circ}}{2 V_{\pi}}
\end{aligned}
$$

where $E_{2}(t)$ is detected by the photodetector. The photodetector voltage can be estimated as

$$
\begin{aligned}
V(t) & =r Z_{\mathrm{PD}} G_{\mathrm{PD}} E_{2}(t) \times E_{2}(t)^{*} \\
& =r Z_{\mathrm{PD}} G_{\mathrm{PD}}[A+B \cos \Omega t]^{2}[C+D \cos (\Omega t+\phi)]^{2}
\end{aligned}
$$

where $Z_{\mathrm{PD}}$, and $r$ are the photodetector load impedance and responsivity, respectively, and $G_{\mathrm{PD}}$ is voltage gain of the low-pass filter. Substituting (5) into (7), and simplifying by dropping the terms $(1) /(4) M^{2} B^{4} \ll A^{4}$ and $(1) /(8) M^{2} B^{4} \ll 2 M A^{2} B^{2}$, the dc component of $V(t)$ can be approximated as

$$
\begin{aligned}
V_{\mathrm{DC}} \approx & r Z_{\mathrm{PD}} G_{\mathrm{PD}} \\
& \times\left[A^{4}+\frac{1}{2}\left(1+M^{2}\right) A^{2} B^{2}+2 M A^{2} B^{2} \cos \phi\right] \\
= & \frac{1}{4} G Z_{\mathrm{PD}} P_{\circ}\left[1+\frac{\pi^{2}\left(1+M^{2}\right) Z_{\mathrm{in}} P_{\mathrm{RF}}}{4 V_{\pi}^{2}}\right] \\
& +\frac{\pi^{2}}{4 V_{\pi}^{2}} G M Z_{\mathrm{PD}} Z_{\mathrm{in}} P_{\mathrm{o}} P_{\mathrm{RF}} \cos \phi
\end{aligned}
$$

where the factor $G$ is defined as $G=r G_{\mathrm{PD}} L_{1} L_{2}$. It is evident from (8) that the photodetector dc voltage includes frequency-dependent functions $M$ and $\phi$. Therefore, it can be used to measure the input RF frequency $\Omega$.

\section{PHOTONIC IFM DEMONSTRATION}

Having established a relationship between the dc output of the photodetector and RF input frequency, it should now be possible to demonstrate frequency measurement. However, as each parameter in (8) is known, except $M$ and $\phi$, the RF path must first be empirically characterized.

\section{A. RF Path Characterization}

To predict the dc voltage of the photodetector by (8), it is necessary to have the absolute magnitude response of the RF path $(M)$ and phase response of the RF path relative to the optical path $(\phi)$ as a function of frequency $\Omega$. Due to dispersive nature of the co-axial cable, the frequency dependence of $M$, and $\phi$ will be nontrivial, and must be measured empirically.

The system was configured as shown in Fig. 2. The laser wavelength and power were set to $\lambda_{1}=1550 \mathrm{~nm}$ and $P_{\circ}=$ $11.7 \mathrm{~mW}$. The factor $G$ was calculated to be 1.2 and the RF signal generator output power was set to $20 \mathrm{~mW}$ which resulted in $P_{\mathrm{RF}}=8 \mathrm{~mW}$ as there was 4-dB loss in the Wilkinson power divider and RF cables. The input impedance of both MZMs 


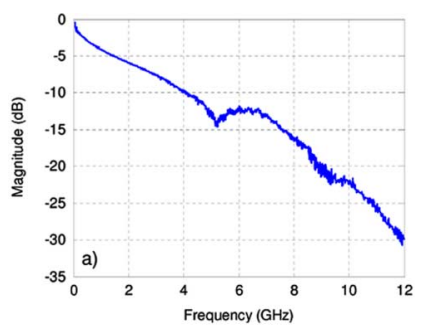

(a)

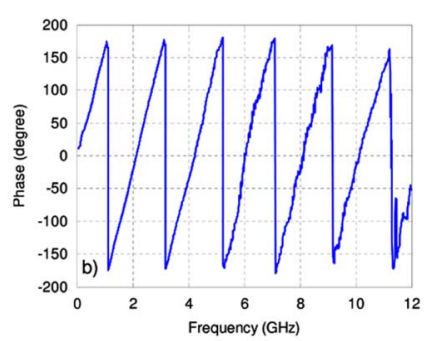

(b)
Fig. 3. (a) Absolute magnitude response $(M)$, and (b) phase response of the RF path relative to the optical path $(\phi)$.
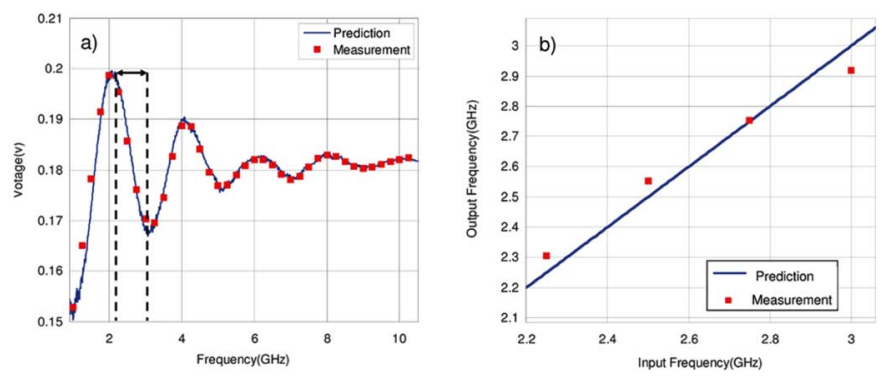

Fig. 4. (a) Measured and predicted photodetector dc voltage. (b) Measured frequency versus input frequency.

$\left(Z_{\text {in }}\right)$ and the output impedance of the photodetector $\left(Z_{\mathrm{PD}}\right)$ were $50 \Omega$. The $V_{\pi}$ of the both MZMs was $5 \mathrm{~V}$.

A vector network analyzer (VNA) was used to characterize the RF path. To measure the phase response of the RF path relative to the optical path $(\phi)$, Port 1 of the VNA was connected instead of the RF signal generator and Port 2 was connected to the output of the photodetector. The VNA was then calibrated with the two ends of the co-axial cable replaced by matched loads. The cable was returned while the input of MZM1 was terminated in a matched load. The VNA then measured the phase response of the RF path with respect to the optical path. The forward transmission amplitude response of the RF path $(M)$ was also measured using the VNA.

Fig. 3 shows the measured magnitude of the forward transmission of the RF path and phase of the RF path relative to the optical path. The magnitude response decreases with increasing frequency as expected. A resonance at $5.5 \mathrm{GHz}$ is evident which can be attributed to the onset higher order modes. The cable was stabilized as the resonance frequency could vary due to vibrations. The phase response is almost linear over the whole band neglecting minor deviations for frequencies higher than $5.5 \mathrm{GHz}$ due to the impedance loading of higher order modes. Fig. 4(a) and (b) shows that the amplitude and phase of the RF path are well-behaved below $5 \mathrm{GHz}$, where the relative phase is almost linear with frequency. The system will thus be suitable for frequency measurement in this frequency range.

\section{B. IFM Characterization}

Having established all of the parameters in (8), the operation of the IFM system can now be demonstrated. The system was configured as depicted in Fig. 2 with the parameters defined in Section IV-A. The dc voltage was measured as a function of
RF input frequency as presented in Fig. 4(a). Clear oscillatory behavior is evident as predicted. The response predicted using (8) is also presented in Fig. 4; excellent agreement is evident.

To perform frequency measurement, a look-up table was generated using (8) and the empirically measured RF cable magnitude and phase response $(M$ and $\phi)$ relating measured dc voltage to input RF frequency as depicted in Fig. 3(a). This look-up table was then used to find the input frequencies that correspond to measured dc outputs. Due to the oscillatory nature of (8), unambiguous frequency measurement is only possible within each half period of Fig. 4(a). Restricting the frequency mapping to one half period $(2.2-3 \mathrm{GHz})$, unambiguous frequency measurement can be demonstrated. The measured frequencies in the 2.2to 3-GHz band are presented in Fig. 4(b).

Fig. 4(a) shows that oscillations in (8) can be detected up to about $10 \mathrm{GHz}$. Beyond this frequency, losses in the RF cable become excessive damping the oscillations. The RF cable in this demonstration was over $5 \mathrm{~m}$ long to match the length of the optical fiber patch cords. The RF losses could be reduced by making both optical and RF paths shorter. An integrated system with path lengths in the order of millimeters would significantly improve the upper limit of frequency measurement and also minimize sensitivity to vibration and thermal fluctuations. The fractional bandwidth could be extended by decreasing the relative delay $(\tau)$ between the RF path and the optical path which will increase the period of oscillation in (8) and Fig. 4(a), but would reduce the gradient and hence the sensitivity.

We are currently investigating the sensitivity that can be achieved using this approach. In particular, phase-locked loops are being explored to enhance the signal-to-noise ratio.

\section{CONCLUSION}

A simple technique to achieve photonic frequency measurement using low-cost low-frequency photodetectors is proposed and demonstrated with frequency measurement up to $10 \mathrm{GHz}$. Options to increase the bandwidth of measurement bands at the expense of sensitivity have also been identified.

\section{REFERENCES}

[1] H. Gruchala and M. Czyzewski, "The instantaneous frequency measurement receiver in the complex electromagnetic environment," in Proc. Int. Conf. Microwave, RADAR, and Wireless Communications (MIKON 2004), May 2004, vol. 1, pp. 155-158.

[2] R. B. Sanderson and J. B. Y. Tsui, "Instantaneous frequency measurement receiver with bandwidth improvement through phase shifted sampling of real signals," U.S. Patent 5109188, Apr. 28, 1992.

[3] M. Aikawa and H. Oqawa, "Double-sided MICs and their applications," IEEE Microw. Theory Tech., vol. 37, no. 2, pp. 406-413, Feb. 1989.

[4] S. Kumar, A. Mohammadi, and D. Klymyshyn, "A direct 64QAM modulator suitable for MMIC applications," Microw. J., vol. 40, no. 4, pp. 116-122, Apr. 1997.

[5] L. V. T. Nguyen and D. B. Hunter, "A photonic technique for microwave frequency measurement," IEEE Photon. Technol. Lett., vol. 18, no. 10, pp. 1188-1190, May 15, 2006.

[6] A. J. Seeds and K. J. Williams, "Microwave photonics," J. Lightw. Technol., vol. 24, no. 12, pp. 4628-4641, Dec. 2006.

[7] R. A. Minasian, "Photonic signal processing of microwave signals," IEEE Trans. Microw. Theory Tech., vol. 54, no. 2, pp. 832-846, Feb. 2006.

[8] J. Capmany, B. Ortega, and D. Pastor, "A tutorial on microwave photonic filters,” J. Lightw. Technol., vol. 24, no. 1, pp. 201-229, Jan. 2006. 